\title{
Is Anti-Gliadin Antibody Pathogenic in Gluten Ataxia? Analysis using Rat Cerebellar Slices and Patch-Clamp Recording
}

\section{Hiroshi Mitoma ${ }^{*}$, Kazunori Nanri² and Hidehiro Mizusawa ${ }^{3}$}

${ }^{1}$ Department of Medical Education, Tokyo Medical University, Tokyo, Japan

${ }^{2}$ Department of Neurology, Tokyo Medical University Hachioji Medical Center, Tokyo, Japan

${ }^{3}$ Department of Neurology and Neurological Science, Graduate School of Medical and Dental Sciences, Tokyo Medical and Dental University, Tokyo, Japan

\begin{abstract}
The significance of autoantibodies associated with neurological symptoms has been the focus of interest. Recent studies emphasized the pathogenic role of anti-gliadin antibody in gluten ataxia, an important disease of autoimmune cerebellar ataxia. To examine whether autoantibodies, including anti-gliadin antibody, play a pathogenic role, we analyzed the effects of CSF samples obtained from a Japanese patient with gluten ataxia on cerebellar synaptic transmission. Patch-clamp recordings were prepared from cerebellar Purkinje cells, the output cells from the cerebellar cortex, in mice cerebellar slices. The CSF (diluted 1:100) had no effects on the excitatory postsynaptic currents, and did not affect the release mechanisms of glutamate. These results do not support an idea that CSF autoantibodies, including anti-gliadin antibody, interfere cerebellar synaptic function so as to develop ataxia.
\end{abstract}

Keywords: Autoantibodies; Ataxia; Gluten ataxia; Anti-gliadin antibody; Synaptic dysfunction

\section{Introduction}

The significance of autoantibodies associated with neurological diseases has been the focus of discussion. In most of autoimmune neurological disorders, it is not clear whether the antibody acts as a pathogenic agent causing symptoms, or it is produced as a result of cell-mediated autoimmune responses against neural structures. To confirm the pathogenic role of autoantibodies in the development of neurological symptoms, the following three criteria need to be demonstrated; 1) Antibodies access the antigens (accessibility), 2) antibodies impair signal flow in neural circuits, which could cause the appearance of neurological symptoms (pathogenic action), and 3) passive transfer of antibodies can mimic these neurological symptoms (passive transfer) [1]

Patch-clamp recording of rat brain slices preparations for measurement of postsynaptic current is a useful method for examination of changes in signal flow in neural circuits (pathogenic action) for two reasons. First, the sensitivity to changes in synaptic transmission is high. Deficits in ion channels, release mechanism of the transmitter, and receptor sensitivity can be detected as changes in postsynaptic current. Second, the neural circuits are maintained in slice preparations. Using this method, we reported previously that autoantibodies to GAD (GAD-Ab) played a pathogenic role in the development of neurological disorders [1-3]. GAD is a catalytic enzyme that converts glutamate to GABA, a major inhibitory transmitter. GAD-Ab is associated with Diabetes Mellitus (DM) and autoimmune cerebellar ataxia. GAD-Ab acts on the cerebellar GABAergic interneuron terminals and depresses the release of GABA on Purkinje cells, the cerebellar output cell, resulting in disorganization of the output from the cerebellum to motor control centers. Taken together with passive transfer experiments [4,5], $\mathrm{GAD}-\mathrm{Ab}$ is now established to cause cerebellar ataxia.

Together with GAD-Ab-associated ataxia, gluten ataxia is a major autoimmune cerebellar disease. Hadjivassiliou et al. [6,7] reported a group of patients with gluten sensitivity presenting with ataxia and detectable levels of anti-gliadin $\mathrm{Ab}$. The prevalence of anti-gliadin $\mathrm{Ab}$ among patients with idiopathic sporadic cerebellar ataxia was significantly higher than in patients with spinocerebellar degeneration disease and normal controls [6,7]. Previous immunohistochemical studies have demonstrated the presence of antibodies against Purkinje cells in patients with gluten ataxia [8]. Furthermore, the molecular mimicry between gliadin and synapsin I has been reported [9]. Since synapsin I is an essential protein in the release of the transmitters, the pathogenic role of anti-gliadin $\mathrm{Ab}$ has been implicated. However, the significance of anti-gliadin $\mathrm{Ab}$ is still uncertain.

The present study was designed to elucidate whether autoantibodies, including anti-gliadin $\mathrm{Ab}$ and yet un-identified antibodies, interfere with cerebellar synaptic transmissions to cause pathological symptoms in gluten ataxia. If anti-gliadin Ab elicits synaptic dysfunction, the CSF would depress cerebellar synaptic transmissions. Thus, the effects of CSF, obtained from a Japanese patient with gluten ataxia, were examined using patch-clamp recording in mice cerebellar slices.

\section{Materials and Methods}

\section{Subjects}

The patient was a 51-year-old Japanese woman who developed unstable gait and scanning speech at 45 years of age. The clinical profile was reported in detail in our previous report [10]. The gait disturbance was progressive and at age 49 she could not walk unaided. Limb ataxia was prominent in addition to severe gait ataxia. Family history of ataxia was negative. Serological testing showed serum antigliadin IgA antibody of 42.7 (EU, normal range $<20 \mathrm{EU}$ ), negative anti-gliadin IgG antibody $(<1.0 \mathrm{EU})$, and anti-SS-A antibody was $15.1 \mathrm{U} / \mathrm{ml}$. Other serum autoantibodies (anti-nuclear antibody, antithyroglobulin antibody, anti-TPO antibody, anti-GAD antibody, anti$\mathrm{Hu} /$ Yo antibody) were negative. Genetic tests for SCA types 1, 2, 3,

${ }^{*}$ Corresponding author: Hiroshi Mitoma, Department of Medical Education, Tokyo Medical University, Tokyo, Japan, E-mail: mitoma@tokyo-med.ac.jp

Received May 22, 2013; Accepted September 04, 2013; Published September 06, 2013

Citation: Mitoma H, Nanri K, Mizusawa H (2013) Is Anti-Gliadin Antibody Pathogenic in Gluten Ataxia? Analysis using Rat Cerebellar Slices and PatchClamp Recording. Brain Disord Ther 2:105. doi:10.4172/2168-975X.1000105

Copyright: (C) 2013 Mitoma H, et al. This is an open-access article distributed under the terms of the Creative Commons Attribution License, which permits unrestricted use, distribution, and reproduction in any medium, provided the original author and source are credited. 
$6,7,8$, and 12 and dentatorubral-pallidoluysian atrophy showed no gene abnormalities. Magnetic Resonance Imaging (MRI) showed no atrophy of the cerebellum, But Single Photon Emission Computed Tomography (SPECT) showed reduced cerebellar blood flow. Intravenous Immunoglobulin (IVIg) was administered four times at intervals of 6 months to 1 year, and each infusion induced marked improvement of clinical symptoms (at the third IVIg treatment, the ICARS scores for posture and gait disturbances improved from 19 to $5)$.

The ethics review committee of Tokyo Medical University approved this research, and a signed consent form was obtained from the patient.

\section{Patch-clamp recording of cerebellar slices}

Patch-clamp recording was prepared using cerebellar slices as described previously [1-3]. Brains were removed from 17-23 day-old mice under $\mathrm{CO}^{2}$ anesthesia. The cerebellar vermis was sagittaly sliced at $250 \mu \mathrm{m}$ thickness. For whole-cell recording, the slice was then transferred to a recording chamber attached to the microscope stage. The slice was continuously perfused with Artificial Cerebrospinal Fluid (ACSF) [(in mM): $125 \mathrm{NaCl}, 2.5 \mathrm{KCl}, 2 \mathrm{CaCl}_{2} 1 \mathrm{MgSO}_{4} 1.25$ $\mathrm{NaH}_{2} \mathrm{PO}_{4}, 26 \mathrm{NaHCO}_{3}$, and 20 glucose, bubbled with $95 \% \mathrm{O}_{2}$ and $\left.5 \% \mathrm{CO}^{2}\right]$. Membrane currents were recorded from visually identified Purkinje cells with a patch-clamp amplifier (Axopatch1D, Molecular devices, Sunnyvale, CA) using the whole-cell configuration. Evoked synaptic currents were produced in response to focal stimulation via glass microelectrodes placed within the molecular layer. Parallel fibermediated excitatory postsynaptic currents were identified based on facilitation of second responses to paired-pulse stimuli. To exclude the contamination of inhibitory postsynaptic currents, bicuculline $(5 \mu \mathrm{M})$, a $\mathrm{GABA}_{\mathrm{A}}$ receptor antagonist, was perfused [1-3].

The slices were preincubated in the CSF-containing ACSF (diluted 1:100) for $1 \mathrm{hr}$ before recording. CSF samples obtained from two patients with cervical spondylosis served as the neurological disease control.

\section{Results}

Focal stimulation within the molecular layer induced sufficient activation of parallel fibers to evoke Excitatory Postsynaptic Currents (EPSCs) on Purkinje cells (Figure 1). In general, these EPSCs showed paired-pulse facilitation with relatively short time intervals (less than $\sim 200 \mathrm{~ms}$ ). First, we examined the effects of CSF from the patient on the amplitude of EPSCs in the rat cerebellar slices. Application of the CSF of the patient did not elicit any change in the amplitude of EPSCs (Figures 2 and 3). The percent change in the EPSC amplitude $8 \mathrm{~min}$ after the application was $102.1 \pm 11.0 \%$ (mean $\pm \mathrm{sd}, \mathrm{n}=5$ ), a response similar to that seen after the application of the neurological disease control CSF $(105.0 \pm 10.2 \%, \mathrm{n}=6)$. We also examined the PairedPulse Ratio (PPR), a physiological index of transmitter release. PPR is defined as a ratio of the amplitude of the second EPSC to that of the fist EPSC (S2/S1). Application of CSF had no effect on the PPF (before the application: $165.7 \pm 14.3 \%, 8 \mathrm{~min}$ after the application: $164.5 \pm 16.0 \%$, mean $\pm \mathrm{sd}, \mathrm{n}=5$ ) (Figure 4 ).

\section{Discussion}

Autoimmune cerebellar ataxia is important since early diagnosis and therapy could relieve the symptoms, which is in contrast to spinocerebellar degeneration. The major two forms of the disease are gluten ataxia and GAD-Ab-associated ataxia. The present results showed that the CSF obtained from a Japanese patient with gluten

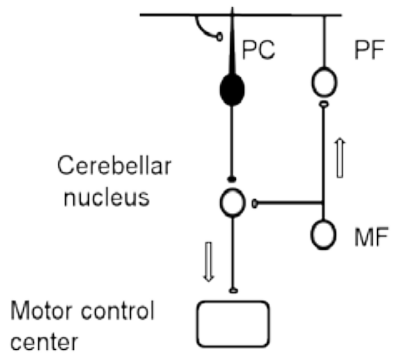

Figure 1: Diagram of neural circuitry in the cerebellar cortex. PC; Purkinje cells, PF; parallel fibers, MF; mossy fibers.

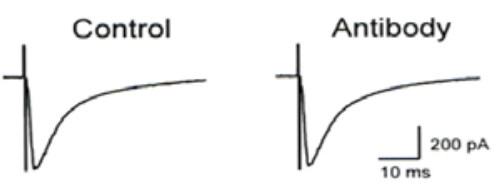

Figure 2: Changes in evoked EPSCs.

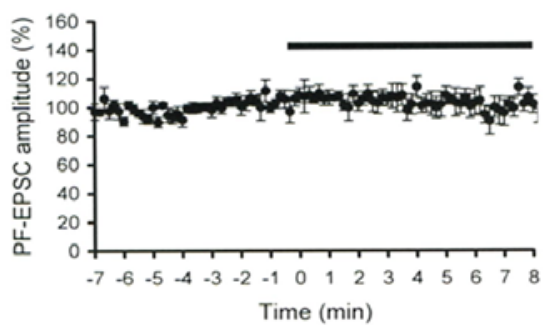

Figure 3: Time course of percent change in the EPSC amplitude before and after the application of the diluted (1:100) CSF. The average and 1sd values of five cells are computed. The CSF was applied by perfusion during the period indicated by the horizontal bar.

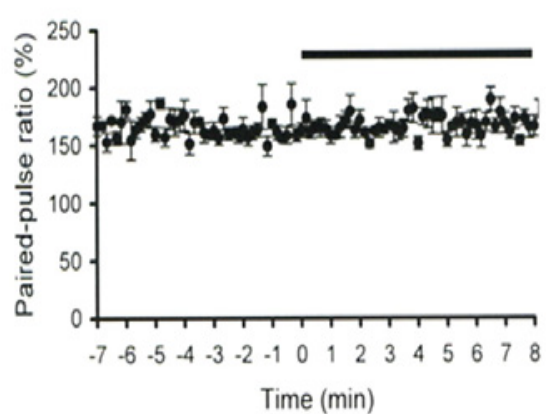

Figure 4: Serial changes in the paired-pulse ratio (S2/S1; ratio of the amplitude of the second EPSC to that of the first EPSC) before and after the application of the diluted (1:100) CSF. The average and 1sd values of five cells are computed. The CSF was applied by perfusion during the period indicated by the horizontal bar.

ataxia did not affect excitatory cerebellar synaptic transmission. We also tested the effects of the CSF on presynaptic transmitter release mechanisms. PPF (the ratio of the amplitude of the second postsynaptic current to that of the first postsynaptic current) is used for assessment of the transmitter release [11]. Impairment of the release mechanism, for example, could result in a decrease in releasable vesicles or a decrease in the release probability, leading to an increase 
in PPF. The present finding indicated that the CSF sample obtained from the ataxic patient failed to change the PPF in EPSCs, suggesting that the CSF did not contain compounds that alter the presynaptic release mechanisms of glutamate. These results do not support an idea that that CSF autoantibodies, including anti-gliadin Ab and yet notidentified antibodies, interfere with cerebellar synaptic transmissions and thus are involved in the development of pathogenic symptoms.

One possible explanation for the lack of effect of the CSF on neurotransmission is that gluten ataxia is a cell-mediated autoimmune response, similar to paraneoplastic cerebellar ataxia. In this case, anti-gliadin antibody might be not pathogenic, but rather a marker of the disease. The alternative explanation is that gluten ataxia is heterogeneous and the present finding of the lack of pathogenic Abs is exceptional. In Japan, the incidence of gluten ataxia is less than in the US and Europe [12]. One reason could be the high consumption of rice as staple food. Furthermore, this patient had an IgA type of anti-gliadin $\mathrm{Ab}$, not IgG type. Thus, the present results could reflect the clinical profile of atypical gluten ataxia.

The present studies do not exclude the possibility that autoantibodies elicit pathogenic actions through mechanisms other than synaptic dysfunction. For example, recent studies show that antitransglutaminase antibodies (anti-TG2, anti-TG3, and anti-TG6-Abs) potentially cause ataxia [13]. Thus, more complicated impairments such as deficits in metabolic pathways might underlie the anti-TG2/3/6Abs- induced development of ataxia.

In contrast to the present results, the GAD Ab plays a pathogenic role in GAD Ab-related ataxia. In in vitro preparations, GAD-Ab interfered with both the synthesis and exocytosis of GABA [6], and resulted in reduced GABA release from GABAergic neurons [1-4]. Furthermore, in in vivo preparations, injection of monoclonal GAD$\mathrm{Ab}$ in the cerebellar cortex diminished cerebellar inhibition of cortical motor excitability $[5,6]$. Taken together with the present results, the autoantibodies in autoimmune ataxia could be classified into two categories; pathogenic Abs and nonpathogenic Abs (i.e., marker Abs). If autoantibodies are pathogenic, immunosuppressive therapy should be applied until the Ab titer is reduced below the detection limits. Thus, characterization of Abs could help in clinical decision making regarding treatment options. For such screening, patch-clamp recording of mice cerebellar slices is a conventional and useful assay technique to examine actions on synaptic transmissions. The technique should be used before the commencement of immunosuppressive therapy, screening using the patch-clamp technique to select the best therapeutic strategy. Further systematic survey is needed to elucidate the physiological properties of anti-gliadin Abs.

\section{Conclusion}

Autoimmune cerebellar ataxia is important because early diagnosis and therapy could relieve the ataxia. Gluten ataxia is a major form of autoimmune cerebellar ataxia. The patch-clamp recording of mice cerebellar slices showed that the CSF obtained from a Japanese patient with gluten ataxia did not elicit any functional impairment of cerebellar synaptic transmission. The results do not support an idea that CSF autoantibodies, including anti-gliadin antibody, interfere cerebellar synaptic dysfunction so as to develop ataxia, at least in this patient.
Further systematic survey is needed to elucidate the physiological properties of anti-gliadin $\mathrm{Ab}$. The patch-clamp technique using mice cerebellar slices could be a conventional and useful assay system to help select effective therapeutic strategies for patients with gluten ataxia.

\section{Acknowledgement}

We are indebted to Prof. Kouichi Hashimoto, Graduate School of Biomedical Sciences, Hiroshima University, and Prof. Masanobu Kano, Graduate School of Medicine, University of Tokyo, for review of physiological data.

\section{References}

1. Mitoma H, Ishida K, Shizuka-Ikeda M, Mizusawa H (2003) Dual impairment of GABAA- and GABAB-receptor-mediated synaptic responses by autoantibodies to glutamic acid decarboxylase. J Neurol Sci 208: 51-56.

2. Ishida K, Mitoma H, Song S-Y, Uchihara T, Inaba A, et al. (1999) Selective suppression of cerebellar GABAergic transmission by an autoantibody to glutamic acid decarboxylase. Ann Neurol 46: 263-267.

3. Mitoma H, Ishida K, Song S-Y, Yamakuni T, Kobayashi T, et al. (2000) Presynaptic impairment of cerebellar inhibitory synapses by an autoantibody to glutamate decarboxylase. J Neurol Sci 175: 40-44.

4. Manto MU, Laute MA, Aguera M, Rogemond V, Pandolfo M, et al. (2007) Effects of anti-glutamic acid decarboxylase antibodies associated with neurological diseases. Ann Neurol 61: 544-551.

5. Manto MU, Hampe CS, Rogemind V, Honnorat J (2011) Respective implications of glutamate decarboxylase antibodies in stiff person syndrome and cerebellar ataxia. Orphanet J Rare Dis 4: 3.

6. Hadjivassiliou M, Grünewald RA, Chattopadhyay AK, Davies-Jones GA, Gibson A, et al. (1998) Clinical, radiological, neurophysiological and neuropathological characteristics of gluten ataxia. Lancet 352: 1582-1585.

7. Hadjivassiliou M, Grünewald RA, Sharrck B, Sanders D, Lobo A et al. (2003) Gluten ataxia in perspective: epidemiology, genetic susceptibility and clinical characteristics. Brain 126: 685-691.

8. Hadjivassiliou M, Boscolo S, Davies-Jones GA, Grünewald RA, Not T, et al. (2002) The humoral response in the pathogenesis of gluten ataxia. Neurology 58: $1221-1226$

9. Alaedini A, Okamoto H, Briani C, Wollenberg K, Shill HA, et al. (2007) Immune cross-reactivity in celiac disease: anti-gliadin antibodies bind to neuronal synapsin I. J Immunol 178: 6590-6595.

10. Nanri K, Okita M, Takeguchi M, Taguchi T, Ishiko T, et al. (2009) Intravenous immunoglobulin therapy for autoantibody-positive cerebellar ataxia. Intern Med 48: $783-790$

11. Saitow F, Suzuki H, Konish S (2005) $\beta$-Adrenoceptor-mediated long-term upregulation of the release machinery at rat cerebellar GABAergic synapses. $J$ Physiol 560: 487-502.

12. Ihara M, Makino F, Sawada H, Mezaki T, Mizutani K, et al. (2006) Gluten sensitivity in Japanese patients with adultonset cerebellar ataxia. Intern Med 45: $135-140$

13. Boscolo S, Lorenzon A, Sblattero D, Florian F, Stebel M, et al. (2010) Anti transglutaminase antibodies cause ataxia in mice. PloS One 15: e9698. 